\title{
EDUCACIÓN PARA LA CARRERA Y GLOBALIZACIÓN: ¿ATRAPADOS EN LAS CONTRADICCIONES SOCIOLABORALES NEOLIBERALES?
}

\author{
CAREER EDUCATION AND GLOBALISATION: CAUGHT UP IN THE SOCIAL \\ AND LABOUR CONTRADICTIONS EXISTING WITHIN NEOLIBERALISM?
}

\author{
Juan Fernández, Sierra*
}

\begin{abstract}
RESUMEN
Los jóvenes están viviendo en una sociedad en la que tenemos planteadas algunas incongruencias difíciles de explicar desde una perspectiva humanista - aumento de las desigualdades en la época de mayor potencialidad productiva, presión hacia la homogeneización cultural en entornos claramente multiculturales, impulso de los nacionalismos paralelamente a la mundialización, etc.- - En este contexto, los orientadores y orientadoras han de repensar su trabajo; no obstante, las presiones económicas, sociales y familiares, les impulsan hacia planteamientos más enfocados al mercado que a la persona. En este artículo, a través del análisis de estas contradicciones y de su reflejo en la OP, se plantean una serie de principios y estrategias para diseñar y poner en práctica proyectos de educación para la carrera que ayuden al estudiantado en su incorporación al marco socioeconómico transnacional en el que le ha tocado vivir desde un concepción de ciudadanía.
\end{abstract}

Palabras clave: Orientación profesional, educación para la carrera, educación sociolaboral, ciudadanía, globalización.

\begin{abstract}
Young students live in a society in which we adults present a number of inconsistencies which are difficult to explain, at least from a humanistic point of view — an increase in inequality at a time of higher production potential, pressure towards cultural homogenisation in clearly multicultural environments, the impulse of nationalism running parallel with globalisation, the pessimism of youth in a society full of opportunities, etc.- - In this context, careers advisers must try to match their work to the needs of the individual, however, economic, social and family pressures push them and direct them

\footnotetext{
* Juan Fernández Sierra es Catedrático de Orientación y Evaluación Educativa. Director del Departamento de Didáctica y Organización escolar y del Grupo de Investigación "Asesoramiento, evaluación y Calidad de la Enseñanza". Sus líneas actuales de investigación son acción psicopedagógica, evaluación y multiculturalidad. Jfsierra@ual.es.
} 
towards approaches which are focused on the market rather than on the person, showing that more and more career advice methods are coming onto the scene with a remarkably consumerist dimension in their notion and in their practice. In this article, the author sets a series of principles and strategies through the analysis of these contradictions and their reflection on careers advice to design and put into practice education projects for studies which contribute to the training of students so that they can take their place, from the concept of citizenship, in the socio-economic trans-national framework in which they have to live.

Key words: careers advice, training for studies, social and work education, citizenship, globalisation

\section{Introducción}

Hay épocas de la historia de la humanidad en las que las contradicciones entre los planteamientos teóricos y las prácticas sociales, siempre sometidos a ciertos grados de tensión, se agudizan de tal manera que, a veces, podemos llegar a pensar que estamos atrapados en ellas sin ver más salida o alternativas que aquellas que nos marcan o indican desde las posiciones hegemónicas. Parece ser que nos encontramos inmersos en uno de esos períodos. En las últimas décadas, paralelamente al desarrollo tecnológico y a las posibilidades que nos ofrecen las nuevas tecnologías de la información, se está forjando un mundo vertiginosamente cambiable en muchos aspectos (sociales, culturales, económicos, políticos, militares, etc.) $\sin$ que las personas y la sociedad cuenten con el tiempo y sosiego necesario para racionalizar e interiorizar nuevas formas de analizar, reflexionar, evaluar, decidir, actuar... Siendo la actividad laboral una de las facetas en más rápida evolución y con más necesidad de readaptación a las situaciones emergentes, y la educación uno de los campos más sensibles y urgente de reorientar, no es de extrañar que al unir ambos aspectos - laboral y educativo-, como ocurre cuando abordamos la orientación profesional en y desde el sistema de enseñanza, nos encontremos con dificultades y paradojas para conseguir una adecuada educación sociolaboral de los y las jóvenes que transitan por el sistema educativo y han de incorporarse en plazo corto o medio a un mundo productivo globalizado.

Con este trabajo pretendemos contribuir a la búsqueda de argumentos y fundamentación teórica, conjugando, interconectando y confrontando algunos análisis que desde la sociología, la antropología, la economía y la educación (Castell, 1997; Wulf, 2004; Estefanía, 1996; Navarro, 1996; Pérez Gómez, 1998) tratan de aportar explicación y comprensión de la sociedad actual, con nuestro conocimiento empírico y experiencia en el campo de la orientación profesional (Fernández Sierra, 2003; Fernández Larragueta, 2002), a fin de plantear vías válidas y útiles para la educación sociolaboral de los y las jóvenes dentro del sistema educativo.

\section{Incongruencias sociales y educación sociolaboral de los jóvenes}

La situación económico-política fruto de la globalización y el pensamiento neoliberal que caracteriza al mundo de finales y principios de siglo ha hecho aflorar o intensificar una serie de contradicciones o paradojas sociales que tienen gran importancia e influencia en los planteamientos educativos en general, y más concretamente de las actuaciones en el campo de la orientación profesional. Entre ellas podríamos destacar: 
El aumento de las desigualdades intra e internacionales en la época de mayor avance tecnológico, y por tanto de mejores posibilidades productivas y de riqueza de la historia de la humanidad. "Nunca como hoy hemos producido tanta riqueza y nunca como hoy ha existido una conciencia tan generalizada de que la pobreza y la miseria son la suerte de la mayoría de los habitantes de nuestro planeta" (Vidal-Beneyto, 2004). Como consecuencia directa de la política económica globalizada al modo neoliberal, ha entrado en crisis el estado de bienestar, una de cuyas principales características, la intervención de los gobiernos para regular el mercado laboral y mantener el pleno empleo se ha visto atacada frontalmente con las medidas de desregulación que pretenden dejar a la iniciativa privada el control total del mercado del trabajo y de los servicios de todo tipo. La pretensión de aumentar los beneficios corporativos se hace frecuentemente por la vía de la disminución de costes, siendo las plantillas y los salarios de los operarios los principales perjudicados. Esto, junto a la maquinización creciente y la introducción de sofisticadas tecnologías, lejos de suponer la liberación de los trabajadores de las tareas más penosas o el aumento de su tiempo libre, se ha convertido, en parte, en una amenaza para la estabilidad laboral. La aparición y aumento del paro, asociada a la lógica del libre-mercado, y la reconversión de derechos básicos como la salud y la educación en proveeduría de servicios ${ }^{1}$, ha traído consigo el aumento de las diferencias sociales. Las desigualdades no dejan de crecer. Desigualdades que se han ido produciendo internamente en cada país, haciéndose cada vez más evidentes las diferencias entre pobres y ricos, apareciendo y extendiéndose una capa amplia de población empobrecida que apenas si subsiste, hasta tal punto que algunos estados europeos parecen aceptar una especie de "tercermundialización" de sus sociedades o una marginación de ciertas capas populares. Pero también aumenta la desigualdad "externamente" ya que cada vez son más pobres los países del llamado Tercer Mundo $^{2}$, provocando, entre otros efectos, el de las migraciones en masa hacia los ricos, a menudo en situación ilegal. Esto, a su vez, acarrea complicados problemas como el rebrote del racismo en las zonas en las que hay un elevado número de inmigrantes, el desarraigo social y cultural generalizado de quienes no tienen más remedio que emigrar, el comercio perverso de personas, los enfrentamientos entre culturas, el fortalecimiento de los integrismos, la superexplotación de los recursos naturales y el trabajo y la explotación infantil (Pérez Gómez, 1998). En este sentido, en las sociedades democráticas, la formación y orientación de los ciudadanos no puede convertirse simplemente en un camino o proceso hacia su "colocación" laboral, sino que debemos proporcionarles una educación sociolaboral que les permita incorporarse a la producción, pero también a la reconstrucción de esos sistemas de producción y de la vida sociopolítica-laboral.

La segunda paradoja que caracteriza la sociedad actual es la presión hacia una homogeneización cultural en contextos cada vez más multiculturales, como reflejo del doble fenómeno de la gran movilidad de personas y el intercambio de información posibilitado

\footnotetext{
1 Incluso en Europa, donde estas políticas de suministro han tenido menos éxito, se han dejado sentir sus efectos, especialmente en las clases menos pudientes. Más evidente son sus efectos en EEUU, y sangrantes en países del Centro y Sudamérica.

2 Los acuerdos de Brettons Woosd, firmados en 1944, "pretendian proteger la democracia y la socialdemocracia, controlando los flujos de capitales y regulando los tipos de cambio (...), el sistema empezó a desmantelarse a principios de los años setenta. Como consecuencia, el sector privado, y especialmente el capital financiero, acapararon más poder todavía. Después de esto hemos asistido, en todo el mundo, a la decadencia del sector público: la debilitación de los sistemas de protección social, el estancamiento o el descenso de los salarios, la prolongación de la semana laboral, el deterioro de las condiciones de trabajo, etc." (Chomsky, 2003: 45-46).
} 
por los medios de comunicación de masas. La gran influencia de éstos, manejados y controlados por grupos económico-políticos con gran poder, tratan de transmitir una cultura hegemónica formalizada y homogeneizada. Por otro lado, el desplazamiento de personas de unos a otros países y culturas por diversos motivos (turismo, intercambio educativo, negocios, etc.) y especialmente los flujos migratorios, hacen que se encuentren los contextos reales con los virtuales o representados. El resultado es una sociedad multicultural que en la vida cotidiana se nos presenta variada y diversa de la que a menudo sólo se proyectan las formas y lo exótico. Pero además es un error pensar que la diversidad cultural es un asunto unido o relacionado solamente con la mayor o menor cantidad de hijos e hijas de inmigrantes que convivan en determinado ambiente o acudan a centros de enseñanza o aulas en el país de acogida. Las "culturas" juveniles son un fenómeno ampliamente extendido en el mundo occidental, bien conocido por los docentes y orientadores que desarrollan su labor con jóvenes estudiantes. La inmigración, en este aspecto, no deja de ser un elemento más a tener en cuenta en la configuración de estas culturas y en la complejidad que ello supone para la acción profesional. Una educación para la carrera adecuada debe procurar capacitar a los y las jóvenes no sólo para entender esta nueva complejidad sino para entremeterse activamente, con rigor, conocimiento y juicio propio en ese marco supranacional e internacional.

La tercera contradicción, íntimamente unida a lo anterior y posiblemente la característica más compleja de la sociedad actual, es que en la era de la mundialización afloran con gran impulso los nacionalismos (Ramonet, 1995). Frente al panorama de homogeneización y uniformidad que trata de imponer el mercado, las personas buscan raíces que las vinculen a su origen, surgiendo movimientos de diversa índole que reclaman su identidad ligada a una cultura, a un territorio, a una religión, a una etnia, etc. La manifestación de este fenómeno está presente continuamente desde formas que enfatizan la propia identidad y cuidan de su conservación frente a la homogeneización, pero que participan al mismo tiempo de los intercambios con otras culturas, hasta propuestas que se justifican a sí mismas en tanto que excluyen o se enfrentan a otras, llegando hasta el integrismo como la versión más radical. De cualquier forma, lo evidente es que, en tanto el nacionalismo ha ido ganando espacio como una manera creíble de articular la identidad, las relaciones laborales y de clase han dejado de ser un referente primordial de movilización colectiva, por lo que la preocupación y el debate político ha ido pasando a esa otra dimensión mientras se han ido olvidando o adormeciendo las reivindicaciones socio-laborales y de justicia social. Mediatizada por estas concepciones, la orientación profesional corre el peligro de ser ejercida en un contexto vacío, imaginario o aparente, que en nada se corresponde con la realidad sociolaboral globalizada con la que se van a enfrentar los individuos y los colectivos profesionales en su futuro próximo.

La cuarta paradoja se constata al observar cómo en la sociedad de las oportunidades, la abundancia y del derroche productivo-consumista, aumenta el pesimismo individual provocando un conformismo social que alcanza cuotas preocupantes entre la juventud. Se extiende la creencia de que no hay más marco social, político y económico que el que se presenta por los poderes hegemónicos, por lo que se hace necesario amoldarse a las condiciones de vida marcadas por el mercado. El convencimiento, con bases más o menos objetivas, de la imposibilidad de cambio y de la inexistencia de alternativas ni utopías, ha llevado a un sector importante de la juventud europea a cotas extremas de pasividad, al tiempo que se manifiestan disconformes y preocupados, social y personalmente, ante su futuro. La re- 
beldía juvenil puede manifestarse activa ante asuntos familiares o sociales, pero se hace pasiva y acomodaticia ante las relaciones laborales. La orientación profesional tiene mucho que hacer en este campo, si es capaz de ir más allá de lo que significa informar de los puestos de trabajo y buscar la adecuación a ellos y quiere implicarse en la educación sociolaboral de ciudadanos autónomos y responsables.

No menos contradictoria resulta la tendencia social, educativa y orientadora que nos impulsa a que, cuanto más avanzamos hacia una sociedad de mayor complejidad política y cultural, manifestemos más obsesión por las soluciones profesionalizadas e individualistas de las problemáticas sociales y personales. No deja de ser llamativa la pretensión o interés de algunos sectores en transmitir a la ciudadanía la idea de que los problemas particulares y públicos deben "ser remediados" por "técnicos externos" con la menor implicación personal o colectiva posible. El avance tecnológico ha supuesto la posibilidad de "cura" a múltiples "padeceres" personales y humanos (desde una enfermedad a la depuración de las aguas o la producción de alimentos...). Pero al mismo tiempo, la idea moderna de "creencia" casi exotérica en la ciencia tecnológica que ha impregnado el pensamiento social, junto a los modos de vida individualista y de constante presión, hedonismo y "premuras" que caracterizan a las sociedades llamadas avanzadas, incitan a la colectividad a abdicar del compromiso y participación en la solución de las contrariedades de los grupos y seres humanos (o sea, a la búsqueda de alternativas políticas), trasladando esa tarea a profesionales especializados, tratando de evadir la implicación directa y la responsabilidad personal y social en dichas soluciones. Esta forma de pensar y actuar, en el campo educativo y concretamente en el de la orientación, se ha dejado sentir especialmente mediante una amplia colonización psicologista - no limitada a una deseable contribución de los conocimientos aportados por esta disciplina a la fundamentación de la teórica y la práctica educativa y orientadora-, sino potenciando las estrategias de intervención clínica "reparadoras" de problemáticas complejas que se entienden y abordan de manera intrapsíquica. Esta psicologización de los problemas sociales y personales (Tadeo da Silva, 2000; Varela, 2000; Hernández Monsalve, 1998) y de la acción orientadora, limita sobremanera la educación sociolaboral y la capacidad real de compromiso actual y futuro de las familias, del profesorado y del alumnado en la toma de decisiones razonadas, al tiempo que convierte en poco útil y limitado el trabajo de los orientadores y orientadoras que así actúan.

En sexto y último lugar, hemos de tomar en consideración la contradicción que puede suponer el hecho de que en un momento histórico de individualismo exacerbado, surjan numerosos movimientos alternativos. La pérdida de ilusión en la democracia participativa como forma de organización política y de avance social junto al marketing consumista y competitivo con el que los medios de comunicación bombardean a los ciudadanos, ha desarrollado en éstos una supervaloración de lo individual. Sin embargo, al mismo tiempo, como reacción a ese super-ego posesivo de mercado (Renduelles, 1998) y a esa situación social económica y política estática sustantivamente, han ido surgiendo una serie de movimientos al margen de las organizaciones sociales, sindicales y políticas tradicionales, formados por colectivos que han ido cristalizando alternativas ante problemas, temas o asuntos variados (ecologistas, feministas, antiglobalización, pacifistas, ONG de diversa índole, etc.) que, aunque minoritarios, han llegado a tener un peso considerable en el entramado social actual. Estos movimientos calan especialmente en la juventud que se incorpora o participa directa o indirectamente en ellos como una forma de solidaridad y defensa de los aspectos 
más débiles del mundo (sociales, personales o naturales). Pero además, situándonos en el campo de la educación para la carrera, les supone un aprendizaje, no sólo por intercambio cultural, sino también como una forma de experiencia laboral y social paralela a su formación académica en la que aprenden a compartir, intercambiar, analizar políticamente la vida y los problemas, trabajar en equipo, responder a objetivos y planes determinados, etc. Su papel como un elemento de primordial importancia en los planteamientos de la orientación profesional, debe ser tenido en cuenta.

\section{Consumo de información, mercado y orientación profesional}

Esta amalgama de contradicciones sociales, cambios en los sistemas de producción y distribución, reestructuración de las relaciones económicas, laborales, políticas, etc., está influyendo sobremanera en los planteamientos de la orientación profesional, de tal forma que, si tradicionalmente ha existido dentro de este campo una importante corriente con una concepción un tanto innatista del ser humano y darwinista de la sociedad que ha basado sus planteamientos e intervenciones en el diagnóstico psicotécnico y el dirigismo, hoy constatamos que aunque los planteamientos teóricos mayoritarios rechazan esos modos de actuar, en la práctica los orientadores y orientadoras, altamente presionados por la sociedad y las familias (a más de por su propio ego profesional), están inmersos en actuaciones fuertemente determinadas por las leyes del mercado, obviando o minimizando la dimensión personal y social de la educación sociolaboral. Esta tendencia, potenciada por el binomio intereses económicos y medios de comunicación, ambos dominados o ligados a las grandes corporaciones capitalistas y grupos de presión hegemónicos, va ganando terreno mediante la construcción de patrones sociolaborales con una alta dimensión consumista. Consumismo que no sólo se refleja en el deseo o ansiedad por la posesión ilimitada de bienes materiales o tangibles, sino que va extendiéndose a todas las manifestaciones de la vida (poder, fama, admiración, presencia, éxito...), alcanzando al pensamiento y actuación profesional de los más diversos campos, pudiéndose observar, en nuestro caso, cómo va conquistando paulatinamente la práctica de la orientación, hasta tal punto que hoy, pienso, podemos hablar de un enfoque, tendencia o sesgo hacia una orientación profesional que podríamos adjetivar de "consumista", en su filosofía y epistemología, en sus propósitos u objetivos y en su práctica.

Considero que en los últimos años la orientación profesional está sufriendo un proceso de involución consumista en cuanto que sus referentes teóricos han basculado desde la persona al mercado, de manera que éste ha pasado de ser un elemento de análisis y destino a convertirse en fuente de iluminación y aporte del sustento de sus finalidades y de su razón de ser. Ha ido asumiendo la ideología de mercado ubicándose bajo la primacía de lo económico, obviando lo personal, lo social y lo político. Podemos observar una corriente aún no institucionalizada en la labor orientadora que parece haber olvidado cualquier planteamiento que no esté imbuido de esa supervaloración de lo previsiblemente pragmático-laboral, analizado bajo la supuesta evolución o desarrollo autónomo de lo tecnológico-productivo, como si ello dependiese de factores o fenómenos que nada tuviesen que ver con las personas, la sociedad, la política o cualquier decisión humana. Esos modos de intervención parecen concebir el mercado desde una especie de visión predeterminista, cuasi-exotérica hacia la que nos veremos arrastrados, en una hipotética dirección más asentada en creencias que 
en datos científicos ${ }^{3}$. La acción orientadora se conceptualiza mediatizada por una representación virtual idílica del mercado, fomentándose modelos reduccionistas que promueven la socialización adaptativa de los jóvenes al status quo. Hemos de absorber y hacer que nuestros estudiantes consuman constantemente ideología de última moda - garantía de construcción de pensamientos y posicionamientos inconsistentes personal y socialmente- para que sean adaptables, flexibles, acomodaticios, compresibles y sumisos al orden económico establecido.

La orientación profesional parece dejarse dominar, subyugar o dirigir en razón del consumo de mercado y del mercado. O sea, trata de guiar a los individuos para que respondan a las demandas de aquél, al tiempo que los orienta para que se preparen para consumir sus ofertas laborales. Se planifica la acción orientadora con el propósito primordial de la satisfacción del mercado y, complementariamente, del consumo de trabajo, real o hipotético, por los estudiantes, futuros operarios, de manera que, a menudo, se actúa en este ámbito como si sólo valiese la pena tomar en consideración, estudiar, informar, orientar o aconsejar en aquello y para aquello que el mercado se prevé que requerirá: se piensa la orientación profesional en aras al consumo de la mano de obra o fuerza de trabajo que el mercado necesita, no en razón de las necesidades humanas que pueden ser cubiertas por el sistema socioeconómico, en el que el mercado es un elemento más; insistimos en el autoempleo o en la importancia del trabajo en equipo porque son factores importantes del desarrollo del capitalismo informacional, no para incidir en la independencia, desarrollo o autonomía personal ni en la cooperación o solidaridad; introducimos la educación para el ocio o para el paro porque en un mercado laboral liberalizado los individuos habrán de pasar tiempo de espera y reciclaje mientras aguardan el paso de un puesto a otro, no como reivindicación o aprovechamiento del tiempo libre y de liberación propia y colectiva; hacemos apología de la formación continua porque los menestrales han de adaptarse, sobrevivir o triunfar en el mercado, no por la conveniencia o el placer de estar actualizados, de ampliar el acervo profesional, de sentir la satisfacción de un trabajo o de un servicio social de calidad bien realizado; la enfocamos pensando dónde podrán colocarse, ser colocados o dónde ganarán más, no en concordancia con las aspiraciones, gustos, anhelos, ilusiones, indeterminaciones, o concepciones de las personas y colectivos sociales. Más que animar a que se conozcan y elijan racionalmente, a los alumnos y alumnas se les pide adaptación y acomodación a lo que se les pueda ofrecer. La profesión se proyecta como una simple manera de supervivencia en el futuro, obviándose la gran importancia que ello tiene, y la indivisible unión e interrelación, en su vida cotidiana

No menos consumista es la práctica cotidiana de la orientación profesional, siendo fácil observar cómo prevalece o toma un peso primordial la acumulación de información y formación "contable", independientemente de su valor educativo. En una época de continua crisis económica, de precariedad en el empleo, de inestabilidad en los puestos de trabajo, de aspiraciones consumistas y de pesimismo social, los orientadores y orientadoras reciben una gran presión de las familias y de la sociedad, induciéndoles a enfocar sus actuaciones, como he reiterado, en función del mercado más que de la persona, de manera que muchos de ellos y ellas han sustituido viejos y obsoletos enfoques dominados por el diagnóstico psi-

\footnotetext{
Es curioso observar cómo mientras los estudios sobre prospectivas económicas y laborales no se atreven a realizar pronósticos fiables más allá de los 3 a 5 años, las prácticas profesionales parecen llevarse a cabo como si hubiese seguridad casi total en el devenir sociolaboral.
} 
cotécnico, por intervenciones y consejos basados en supuestos diagnósticos o prospectivas de mercado, de manera que parece no haber nada que valga la pena estudiar o realizar que no sea en esa dirección (Halliday, 1995; Fernández Sierra, 1999, 2001).

Incluso los orientadores y equipos multiprofesionales sensibles a otras dimensiones más sociales y personales de su labor que desarrollan o colaboran en proyectos o actuaciones en los que tienen en cuenta muchas otras dimensiones, lo hacen de forma segregada de los programas de orientación profesional ${ }^{4}$. Poco o nada se dedica en ellos a que los chicos se den cuenta de la diferencia entre el mundo real en el que les ha tocado vivir y la representación que de él hacen los medios de comunicación; a que comprendan la necesidad de ser capaces de trabajar y plantear actuaciones colectivas, con personas de diferentes culturas, género, clase social, nivel cultural o edad; sean capaces de analizar y posicionarse ante los problemas sociolaborales o políticos, etc.

En resumen, numerosas actuaciones de orientación profesional en los últimos años están olvidando la dimensión social del trabajo y centrándose sólo en la individual, obviando la política y ciñéndose a la técnica, ignorando al ser humano y ensalzando al mercado. El predomino de lo económico sobre lo social y humano se transmite sutil o subliminalmente en la actuación orientadora en el ámbito profesional, la finalidad tácita u oculta es la socialización en una concepción sociolaboral que responda a un concepto de mercado del trabajo precario, aunque presentado bajo el discurso de la flexibilidad y la competitividad. Se desvaloriza la formación y el estudio como medios de satisfacción y crecimiento humano y de valor social. La persona se ignora, el mercado se sobrevalora.

\section{Educar para la carrera en un marco transnacional}

La constatación de los cambios en los modos de producción, en las relaciones laborales e, incluso, en las formas diversas de entender la democracia, no pueden dejar impasibles a los sistemas educativos ni a ninguna de sus funciones. La orientación y apoyo a los jóvenes estudiantes para que se desenvuelvan de manera efectiva por él y para que se incorporen posteriormente a la vida laboral como personas libres, innovadoras y responsables, adquiere una dimensión de mayor calado y complejidad de lo que era tradicional (Fernández Sierra, 1999; Jarvis, 2001; Romero Rodríguez, 2004). Nos encontramos con unas instituciones educativas asentadas en concepciones modernas e incluso premodernas en las que sigue instalado en gran parte un currículum vocacional-neoclásico, la creencia en el valor per se de ciertos conceptos disciplinares, hábitos y conductas que la escuela debe transmitir y una visión utilitarista de los aprendizajes. Esto fomenta una planificación rígida y compartimentada de los contenidos, de los espacios y de los tiempos; una metodología docente demasiada unidireccional, y unos modos de aprendizaje pasivos y poco reconstructivos. Mientras tanto los saberes en las artes, las ciencias y las letras son cada vez más amplios y más complejos, especializados e interrelacionados; la sociedad se va haciendo más diversa en todos

\footnotetext{
4 Muchos orientadores y orientadoras de centro plantean y se implican en proyectos educativos: para la paz, intercultural, para la salud, coeducación, etc., demostrando ser sensibles, estar preocupados y tener una visión mucho más rica del papel de la escuela y de las necesidades formativas de los y las jóvenes, pero no siempre esto lo reflejan o integran en los programas de OP que ponen en marcha (Fernández Sierra, Fernández Larragueta y Rodríguez Fernández, 2003).
} 
los ámbitos, y la vida sociolaboral más indefinida, inestable, flexible y dinámica. No es de extrañar que vivamos profesionalmente con contradicciones y cierta desorientación.

El debate sobre la educación para la carrera no debería centrarse en las necesidades reales o virtuales del mercado ni en las características o rasgo personales en relación al desempeño de una profesión u oficio, sino en lo que necesita la persona (ser individual y social) para poder incorporarse con garantía a una sociedad y a un mundo laboral que se está reconstruyendo, en continua evolución y cambio en sus dimensiones sociales, económicas, culturales, laborales, etc. La orientación ha de debatir sobre las finalidades y principios de actuación que habrían de regir su práctica a fin de responder a las necesidades actuales de los individuos en los aspectos que le son propios (Guichard, 2004). En esta línea, en mi opinión, habríamos de proponernos que, a lo largo del periodo de escolarización, los y las jóvenes tuviesen acceso a cuatro campos o ámbitos que les son imprescindibles para su educación sociolaboral: la comprensión del sistema socio-productivo, el conocimiento del sistema educativo, la preparación para las transiciones y la esfera de la maduración personal.

Respecto al primero, es necesario que las actuaciones orientadoras proporcionen a todos los estudiantes un amplio conocimiento del sistema socio-productivo. Que se percaten del mundo en que han de vivir y trabajar, no sólo se queden con la representación que de él hacen los medios de comunicación. Procurar que se conciencien y entiendan el impacto de los avances tecnológicos en el medio natural y social y, consecuentemente, en los cambios económicos, así como su repercusión en el trabajo y en los modelos ocupacionales. Que lleguen a comprender los conceptos básicos sobre la economía y el trabajo: oferta y demanda, creación de riqueza, libertad de mercado, flexibilidad de plantillas, movilidad en el empleo, responsabilidades y compromisos socio-laborales de los empresarios y de los empleados, papel y función de las organizaciones sindicales y patronales en el sistema democrático y de producción, etc. Informarles y ayudarles a desarrollar estrategias para que puedan conocer los diversos tipos de empleos y ocupaciones locales, regionales, nacionales y europeos; sepan acceder a las ayudas económicas disponibles en el ámbito ocupacional, desde las subvenciones para programas de formación, subsidios de desempleo y sociales, hasta las posibilidades de financiación para la creación de cooperativas y empresas, etc. Pero todo esto habríamos de trabajarlo sin perder de vista que un ciudadano sociolaboralmente educado ha de ser competente para construir su propia opinión sobre las relaciones entre política, economía, sociedad e individuo y ser capaz de tomar sus propias decisiones racional y éticamente.

El segundo ámbito que se ha de tomar en consideración desde la educación para la carrera es el entorno educativo. Posiblemente este sea el menos descuidado desde los programas o proyectos de orientación, no obstante quisiera hacer hincapié en la necesidad de preparar a los estudiantes para que se desenvuelvan con conocimiento y soltura dentro del sistema de enseñanza, reciban información y formación de los caminos y oportunidades que se les ofrecen dentro de él (variedad de estudios, salidas profesionales, ayudas y becas, movilidad nacional e internacional, etc.), pero que además perciban y comprendan la importancia y necesidad de la educación permanente y de la actualización profesional durante la vida adulta. La concepción clásica tan arraigada en nuestros centros de que la consecución de un título dirige hacia una profesión u ocupación laboral determinada y sempiterna, no se corresponde con la realidad que estos chicos y chicas les va a tocar vivir.

El tercer aspecto básico que hemos de trabajar para una apropiada educación sociolaboral es el entramado de las transiciones. Hablar hoy de este tema no es sólo centrarse en el 
paso de un nivel educativo a otro, sino que hemos de considerarlo como un proceso complejo y multidimensional que, aunque tenga momentos más visibles, no por eso es algo puntual ni unidireccional. Ya no se trata sólo del transito de la familia a la escuela, de ésta al instituto, a la universidad, etc. (Gimeno, 1996; Fernández Sierra, 1999; Alfaro, 2004) Los jóvenes de hoy, sin olvidar la importancia de estos aspectos, a lo largo de su vida habrán de "transitar" reiteradamente desde la formación al trabajo y de éste a la primera, cambiar de ámbitos sociogeográficos, moverse de un ambiente cultural a otro, reacomodarse a nuevas estructuras sociales, vivir la reconstrucción permanente de su propia cultura, etc. Todo ello nos obliga a proyectar y poner en escena actuaciones de orientación profesional en una dimensión mucho más ambiciosa y compleja de lo que ha podido ser la habitual información y consejo individualizado a los estudiantes. Ahora necesitan aprender a autodirigirse no sólo en la transición de la vida escolar hacia la vida adulta y la vida laboral, sino entre las diferentes situaciones y circunstancias, para lo que precisan utilizar los resortes disponibles en la comunidad para descubrir y acceder a las posibles ocupaciones y para aprovechar las oportunidades que su formación les brinda, ser capaces de preparar los proyectos que contengan sus planes, seleccionar y manejar información válida y útil para sus intereses ocupacionales y socioculturales, evaluar, elegir y decidir.

El cuarto y esencial ámbito de una adecuada planificación de la educación sociolaboral es la actuación facilitadora de la maduración personal. En realidad, ningún proyecto orientador o educativo tendría justificación razonable en un sistema democrático si no se dirigiera al fortalecimiento, formación, apoyo y liberación del individuo; no obstante, como reiteradamente vengo diciendo, dicha planificación a menudo está más pensada con mentalidad y visión de mercado que de ser humano. Por ello, como siempre y más que nunca, es preciso en estos momentos recordar que las acciones de orientación profesional deben tener como prioridad la capacitación de los y las jóvenes para la toma autónoma de decisiones. Han de aprender a autovalorarse y adquirir conciencia de cuáles son los tipos de actividades y trabajos en los que se encontrarán a gusto y podrán realizar satisfactoria y eficazmente; a conocerse y comprenderse a sí mismos: su fuerza y limitaciones, sus habilidades, sus destrezas, sus cualidades personales, sus potencialidades, sus necesidades, sus aspiraciones, sus aptitudes y valores, etc. Poder ejecutar tareas, tanto de forma independiente como cooperativamente, trabajar individualmente y en equipo con personas de su mismo o diferente edad, sexo, formación y cultura, así como comunicarse y colaborar personal y profesionalmente con ellos; ejercitar efectiva y éticamente la responsabilidad en todos los ámbitos de su vida personal y social; interesarse por las oportunidades futuras; saber defender los derechos individuales y colectivos, participando en la vida social, sindical y política; hacer frente a los cambios que la vida adulta les deparará: independencia, adaptabilidad a diversidad de situaciones, flexibilidad y comprensión de los demás, seguridad y confianza en sí mismos, etc.

\section{Conclusiones}

En unos momentos en los que la educación de la juventud necesita incidir en la formación y reconstrucción de nuevas identidades personales y sociales, haciéndose imprescindible ensanchar su capacidad de comprensión e ineludible potenciar los modelos de análisis y pensamiento, no podemos, desde el campo de la teoría y la práctica orientadora - a pesar 
de que nos sintamos algo desorientados por la ambigüedad e incertidumbre consustancial al mundo actual y a la naturaleza de nuestro labor-, responder con planteamientos y modelos de actuación simplificados y simplificadores que poco concuerdan con la complejidad a la que pretendemos responder. Será altamente difícil lograr, con planteamientos sectorizados, atomizados y ámbitos de acción desconexionados, que los y las estudiantes lleguen a poder comprender el complejo sistema de relaciones laborales, socio-económicas y políticas de nuestros días. Si cada sector profesional se encasilla en sus respectivos "reinos de taifas" (clases, despachos, especialidades, cargos, etc.), en vez de dar pasos firmes y decididos hacia la coordinación de las actuaciones docentes y orientadoras y hacia la integración en los currícula escolares de los conocimientos necesarios para una educación para la carrera amplia (Law, 1996), la escuela se seguirá separando de la vida y las actuaciones que en ella realicemos se convertirán en obligaciones paralelas a la existencia y quehacer de nuestros y nuestras jóvenes. Necesitamos personas no sólo informadas e instruidas, sino formadas y educadas, por lo que no podemos concebir la educación para la carrera como un programa anexo, no se sabe cómo, a las demás actividades curriculares del sistema educativo, sino que ha de presentarse integrado en los currículos, planteamiento que requiere basarlo, al menos, en cuatro principios pedagógicos: corresponsabilidad interprofesional, enfoque interdisciplinar, perspectiva educativa y proyección vital y para la vida.

\section{Referencias bibliográficas}

Alfaro Rocher, J. (2004). "Diagnóstico en Educación y transiciones". Revista Española de Orientación y Psicopedagogía, 15 (1), 67-88.

Castells, M. (1997). La era de la información. Economía, sociedad y cultura (I): La sociedad red. Madrid: Alianza.

Chomsky, N. (2003). Dos horas de lucidez. Barcelona: Ediciones Península.

Estefanía, J. (1996). La nueva economía: la globalización. Madrid: Octubre.

Fernández Larragueta, S. (2002). La acción psicopedagógica en los institutos de educación secundaria. Análisis de su reconstrucción y procesos de socialización. Tesis doctoral. Universidad de Almería.

Fernández Sierra, J. Fernández Larragueta, S. y Rodríguez Fernández, A. (2003). Práctica de la acción psicopedagógica en Almería. Almería: Servicio de Publicaciones de la Universidad de Almería.

Fernández Sierra, J. (1999) "Orientación y transición en educación secundaria. Del dirigismo a la integración curricular”. Cuadernos de Pedagogía, 282: 62-67.

Fernández Sierra, J. (1999). "L'Orientation professionnelle intégrée dans les programmes scolaires: de la socialisation à L'éducation”. L'Orientation Scolaire et Professionnelle, 28 (2), 327-342.

Fernández Sierra, J. (1999). "Orientación y transición en Educación Secundaria. Del dirigismo a la integración curricular”. Cuadernos de Pedagogía, 282, 62-67.

Fernández Sierra, J. (2001). "La orientación profesional en la enseñanza obligatoria: algunas contradiciones educativas". En Santana Vega. L. (Coord): Trabajo, educación y cultura. Un enfoque interdisciplinar. Madrid: Pirámide.

Fernández Sierra, J. (2003). Estudio de la evolución del trabajo psicopedagógico en los institutos de secundaria obligatoria. Informe de Investigación. Universidad de Almería.

Gimeno Sacristán, J. (1996). La transición en la educación secundaria. Madrid: Morata. 
Guichard, J. (2004). "Se faire soi”. L'Orientation Scolare et Profesionale, 4, 499-533.

Halliday, J. (1995). Educación, gerencialismo y mercado. Madrid: Morata.

Hernández Monsalve, M. (1998): "Dilemas en psiquiatría: entre el consumismo, la eficiencia y la equidad". Genealogía del poder, Neoliberalismo versus Democracia, 29: 264-281.

Jarvis, P. S. (2001). "Fórmula para el éxito en la construcción de la carrera profesional". Revista Española de Orientación y Psicopedagogía, 21, 5-14.

Law W. B. (1996) "Careers education in a curriculum". In Watts, A. G.; Law, B.; Killeen, J. (Eds.) (pp. 210-232). Rethinking Careers Education and Guidance. London: Routledge.

Navarro, V. (1996): "Neoliberalismo, desempleo, empleo, y estado de bienestar". Sistema, 134, 27-63.

Pérez Gómez, A. I. (1998). La cultura escolar en la sociedad neoliberal. Madrid: Morata.

Ramonet, I. (1995). "Pensamiento único y nuevos amos del mundo". En Chomsky, N. e Ramonet, I. Cómo nos venden la moto. Barcelona: Icaria, 55-102.

Renduelles, G. (1998). "La Psiquiatría como mano invisible del orden neoliberal”. In Pastor, Renduelles y Valera (Eds.) (pp. 197-217). Neoliberalismo vs democracia. Madrid: La Piqueta.

Romero Rodríguez, S. (2004). “Aprender a construir proyectos profesionales y vitales". Revista Española de Orientación y Psicopedagogía, 15 (2), 337-353.

Tadeus Da Silva T. (2001). Las pedagogías psicológicas y el gobierno del yo en tiempos neoliberales. Sevilla: Publicaciones MECEP.

Varela, J. (2000). "El triunfo de las pedagogías psicológicas". En Tadeu da Silva (Coord) Las pedagogías psicológicas y el gobierno del yo en tiempos neoliberales. Sevilla: Publicaciones M.C.E.P. 75-82.

Vidal-Beneyto, J. (2004). “Caos y gobernación del mundo”. El país (18/09/04).

Wolf, Ch. (2004). "Anthropologie historique. Nouvelles perspectivas sur les fundamentes et les conditions de l'éducation". L'orientation Scolaire et Professionnelle, 33 (4), 571-586.

Fecha de recepción: 03-06-05

Fecha de revisión: 06-02-06

Fecha de aceptación: 17-10-06 